\title{
VoIP and Voice over LTE
}

\author{
Sridevi \\ Assistant Professor, Department of computer Science, Karnatak University, Dharwad.
}

\begin{abstract}
The LTE system was optimised for high data rates and high quality voice services from the beginning of its development. Since LTE represents an all-IP mobile communication system, i.e. a system that is only concerned with the reception and transmission of packet data from and to the user, traditional circuit switched voice and messaging capabilities are not supported. This research paper describes two possible approaches that enable the use of voice and SMS services in LTE: the utilisation of VoIP techniques and the use of the existing $2 G$ or $3 G$ mobile network infrastructures. Furthermore, an insight on the problems and concerns of their techniques is given.
\end{abstract}

Keywords: VOIP, LTE, 3GPP, IP multimedia, etc

\section{Introduction}

Even though mobile packet data transmission grew slowly in the beginning, its tremendous growth made evident that the existing mobile communication systems and networks are not suited to support both needed capacity and a constantly high quality of service (QoS) for their users at the same time. The previously mentioned user-amount-triggered improvement and evolution of data rates was overcome by the vast number of those very users. Networks soon became oversaturated, causing additional costs for operators and providers, which countered the problem with additional elements and components in the means of infrastructure. The real solution of this phenomenon and system situation is provided by a technology evolution, which is based on the existing, modern mobile communication standards, but only uses their beneficial characteristics and components which are elementary for operation. Such a technology is introduced as 3GPP Long Term Evolution, which is a real mobile packet-data-oriented communication system and standard. LTE covers the evolved system requirements in terms of sufficient capacity, increase of data rates and bandwidth, as well as the support of exclusively the packet switched domain. This ensures the simplicity of the system, which also positively reflects on possible transmission delays, i.e. directly on the QoS. Furthermore, the LTE technology enables a more efficient utilisation of the existing and new infrastructure, as well as of the air interface, including the frequency spectrum. With the satisfaction of these requirements, the LTE system surpasses all previous mobile communication systems in the majority of functions, services and mechanisms.

\section{Voice and Messaging Basics in LTE}

Despite of the rapidly growing mobile data traffic, voice calls and SMS messaging still comprises a large percentage of a mobile operators' revenue. Due to this fact, enabling voice and messaging services in LTE became one of the main priorities in LTE development. Several techniques that allow the use of mentioned services, which have been introduced and tested on the LTE air interface, are divided into two approaches:

- Treating voice and messaging processes as data services. This approach is based on Voice over IP techniques and realized through a separate network (IP multimedia subsystem, i.e. IMS) or a third party service provider (e.g. Skype).

- Reverting to mobile networks of previous generations (e.g. GSM, WCDMA or CDMA). This approach allows the use of traditional circuit switched voice calls and SMS messaging.

\subsection{Voice over IP Approach in LTE}

The utilisation of VoIP as LTE's voice bearer is causing both sympathy and aversion of mobile operators. Although it is the simplest approach which does not require many changes of the existing infrastructure, the certain lack of standardisation and problems with specific scenarios (e.g. roaming) steer the operators' opposition. However, the following two solutions that use the VoIP interface have been accepted and implemented into LTE.

\subsubsection{Partnership with existing VoIP service providers}

One possible implementation is realised through the support of existing VoIP services such as Skype. A similar partnership would bring minimal changes to the existing system, as the communication between the external VoIP server and the UE takes place on a data transmission level. The communication concept is based on a two-stage interaction between the two communicating UEs and the external VoIP server in between. To set up a call, the LTE UE transmits VoIP signaling messages (in form of normal packet data) to the VoIP server, which then exchanges similar messages with the other UE (either packet data or a circuit switched signal stream). A block diagram of the system is given on Figure 1. 


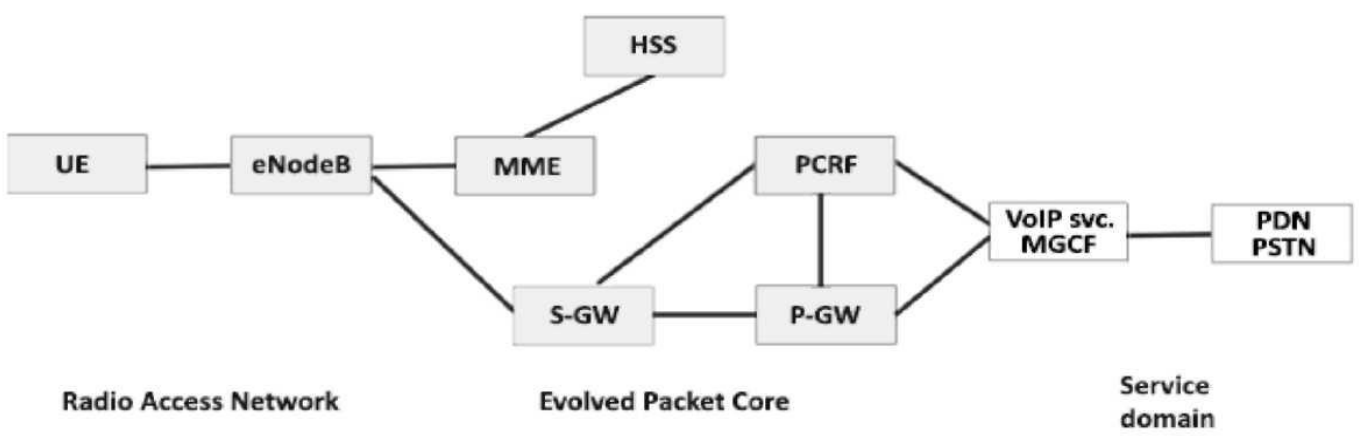

Figure 1: The structure of external VoIP subsystems in EPS

To keep the Quality of Service (i.e. QoS) at a constant high level, a new process is introduced, named Policy and Charging Rules Function (in further text PCRF). This function receives and analyses VoIP signaling messages sent by the communicating UEs and applies the required number of signal bearers to improve the data transmission (LTE side), i.e. voice call transportation (circuit switched side). The conversion of these data streams is handled by media gateways as part of the external VoIP system, which enable the communication between an LTE based UE and UEs based on mobile networks of previous generations. One major omission of this implementation are the so called Fallback Techniques, which would allow the continuation of VoIP voice calls in the event of coverage loss, through "falling back" to $2 \mathrm{G}$ or $3 \mathrm{G}$ mobile networks.

\subsubsection{The IP Multimedia Subsystem}

The IP multimedia subsystem (IMS) acts as a standalone network, interconnected with the packet core of LTE and the packet switched domains of GSM and UMTS. Since it is a separate communication system, implementing it means adding a whole new part to the existing infrastructure. Before this technique was considered for the voice and messaging services in LTE, it was a small project with the main goal to improve the characteristics of $3 \mathrm{G}$ mobile networks. As it introduced additional complexity in contrast to only few improvements, the project was frozen in 2002. It was reassessed in the beginnings of LTE development and immediately determined to be the long-term solution for its voice and messaging requirements. The most important component of IMS is known as the Call Session Control
Function (CSCF), which is distributed in three specific subfunctions:

- The Serving CSCF (S-CSCF), managing the UE and the signaling for incoming or outgoing calls.

- The Proxy CSCF (P-CSCF), managing the signaling of the IMS, compressing and encrypting the signaling messages to reduce the network load and provide additional security. It also communicates with the PCRF, granting a high QoS.

- The Interrogating CSCF (I-CSCF), managing the incoming signaling messages between the other UE and the IMS.

These three sub-functions are interconnected with signaling protocols called Session Initiation Protocols, which are responsible for the intercommunication between IMS elements and carry out UE transmission requests. Furthermore, these protocols are used to expand the system with additional services such as voicemail, located in Application Servers (AS). Another important component is the so called IMS Media Gateway, a version of the VoIP media gateway specifically tailored to the requirements of the IMS system, which uses Media Gateway Control Functions (MGCF) to communicate with circuit switched networks, i.e. PSTNs (Figure 2). Since the MGCFs control the conversion of signaling messages, they are managed by the previously mentioned S-CSCF.

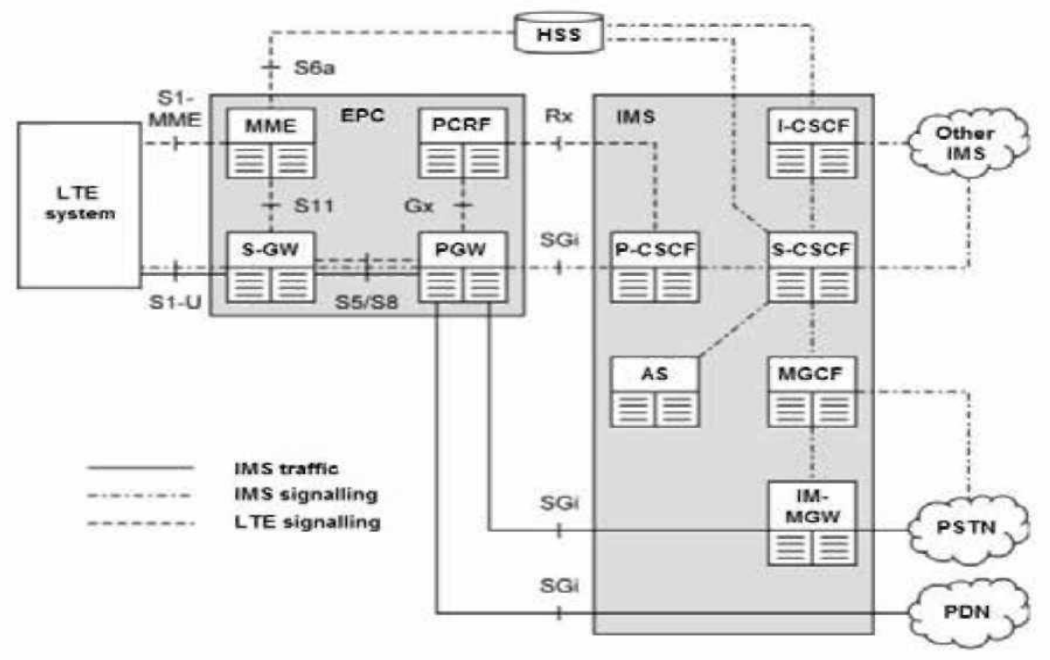

Figure 2: The IMS system architecture 


\section{International Journal of Science and Research (IJSR) \\ ISSN (Online): 2319-7064}

Index Copernicus Value (2013): 6.14 | Impact Factor (2014): 5.611

The system layout designed for voice calls is also very suitable for text messaging, as no major additions have to be made. The only extension is manifested as the IP Short Message Gateway (i.e. IP-SM-GW) which connects the IMS with the standard SMS network components. Those components are mainly the SMS Interworking MSC for outgoing messages and the SMS Gateway MSC for incoming messages (Figure 3).

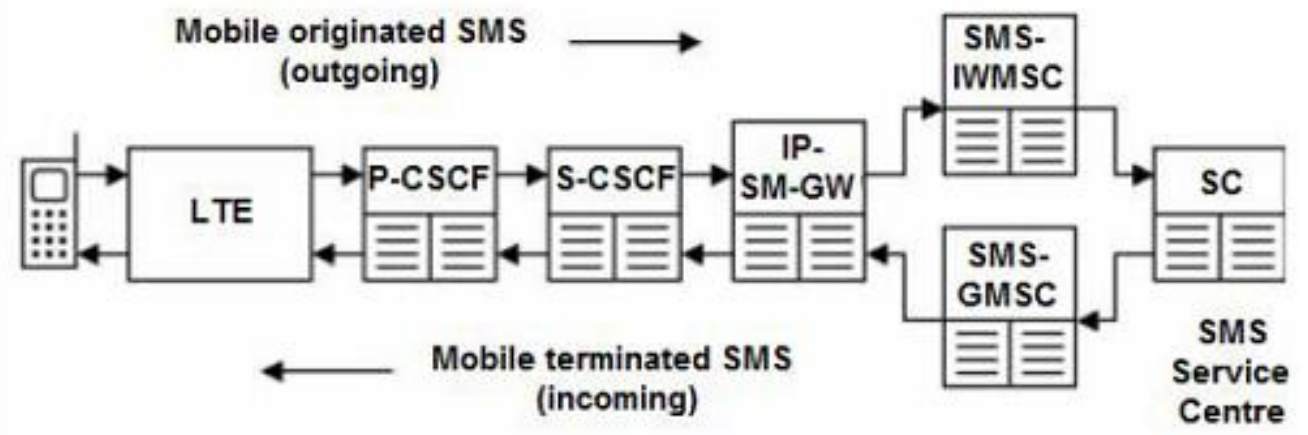

Figure 3: SMS messaging using the IMS system

The utilisation of IMS also introduced the need of a new user-definition system; two new identification elements have been adopted. First, the Private Identity, similar to the IMSI in circuit switched networks, serves to identify the UE to the IMS. Second, the Public Identity, similar to mobile phone numbers or email addresses, serves to identify the UE to the outside world (i.e. beyond the IMS). Both elements are stored in the IP Multimedia Service Identity Module, abbreviated ISIM, to imply the parallels to the previously used USIM.

\section{Fallback to Other Mobile Networks}

The second approach to enable voice and messaging services in LTE was introduced as an interim solution until the new IMS structure is fully integrated in the existing infrastructure. It is based on the possibility to hand over users between different mobile networks without many additions in the existing systems and is fully relying on the voice capabilities of these networks. The transmission of text messages, however, is based on specific principles of the individual techniques.

\subsection{Circuit Switched Fallback}

The Circuit Switched Fallback technique (in further text CS fallback) is the widely accepted solution for voice and messaging services within LTE. It uses a fallback function to revert users (i.e. the calls of a UE) from the LTE network to circuit switched networks (GSM, WCDMA) and viceversa. To support that fallback function, the system architecture is built on top of so called $2 \mathrm{G}$ and $3 \mathrm{G}$ interoperation architectures. To use these procedures, a new network element has to be added to the LTE system: the Mobile Switching Centre server (MSC) which communicates with the Mobility Management Entity (MME) of LTE's packet core system (Figure 4). When a UE initiates a voice call (i.e. a Mobile Terminated Call takes place), it first sends a combined EPS/IMSI attach request to the MME which indicates whether a fallback is possible. If the request is accepted, the MME issues a location update that informs the circuit switched network of the new UE's position, simultaneously searching for a suitable MSC. After additional steps of identification and security, the UE registers to the MSC by sending out so called SGs messages, using it as a gateway to connect to a circuit switched mobile network. At this moment, the eNodeB base station starts the packet handover from LTE to the chosen network, triggering an incoming call to the target UE and setting up a call.

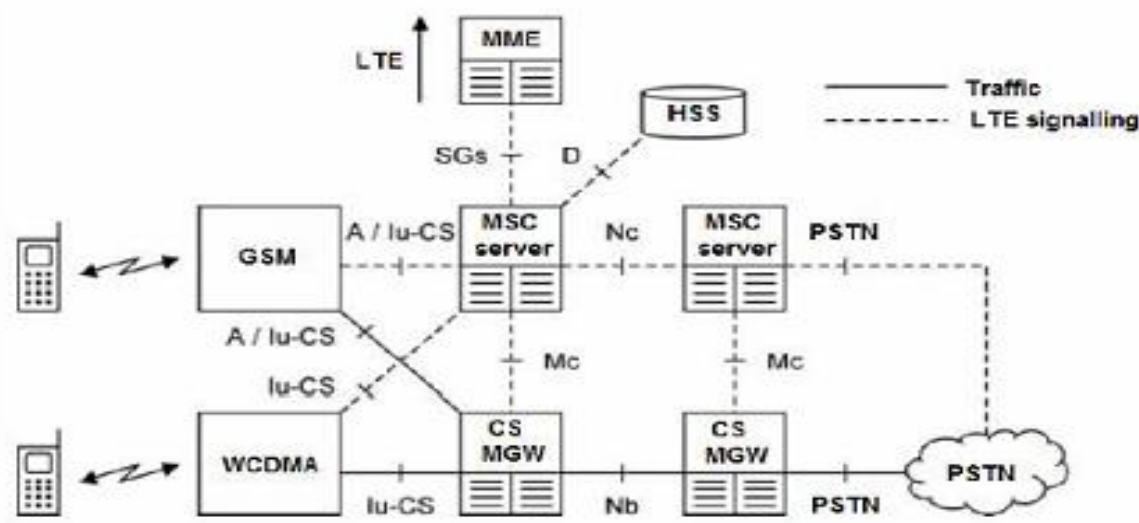

Figure 4: Circuit switched fallback architecture, attach request route

The procedure of an incoming call (i.e. Mobile Originated Call) can be seen as exactly reverse to the outgoing call scenario. When the calling UE sends a voice call request to the eNodeB base station, it starts the packet handover,

\section{Volume 4 Issue 12, December 2015}




\section{International Journal of Science and Research (IJSR) \\ ISSN (Online): 2319-7064}

Index Copernicus Value (2013): 6.14 | Impact Factor (2014): 5.611

matching the previously described call establishment again. procedure. After the call has ended, the UE connects to LTE

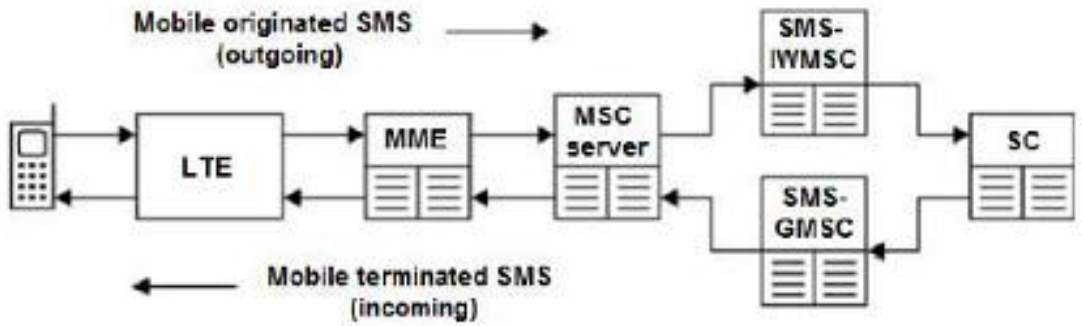

Figure 5: SMS messaging using the SMS over SGs technique

The CS fallback technique requires only minor upgrades of the existing system infrastructure, but introduces a number of drawbacks as well. With these issues mostly being service degradations, its acceptability is questionable. One of these issues is the implementation of SMS messaging. Due to the large number of reselections and handovers between LTE and GSM or WCDMA in the event of sending large amounts of SMS messages, the CS fallback process was classified as inefficient. This issue is encountered with the proposal of a technique known as SMS over SGs, which can be applied to the existing interface. The messages are therefore incorporated into the signaling messages sent to the MME, which forwards them to the MSC (Figure 5). This process is an equivalent to the technique for SMS messaging used in the IMS structure.

\subsection{Voice over LTE via Generic Access}

Another fallback technique suitable for LTE is called the Voice over LTE via Generic Access method (i.e. VoLGA), an industry based initiative introduced in 2009. As it is based on the easy-to-implement 3GPP Generic Access Network architecture (GAN), which was developed to support circuit switched services such as SMS messaging in an IP-based network, it quickly gained attention and became one possible candidate for LTE's voice and messaging requirements. The GAN techniques enable the UE to register to a GSM network through a WLAN connection, allowing the use of its services. In the VoLGA implementation, however, the traffic is routed through the LTE network instead. The only hardware addition to the existing network is an interface known as the VoLGA Access Network Controller (VANC). This element behaves as an extra network node which is connected to LTE's core network through the PDN gateway, its main function being the intersystem handover. A block diagram of the VoLGA architecture is given on Figure 6.

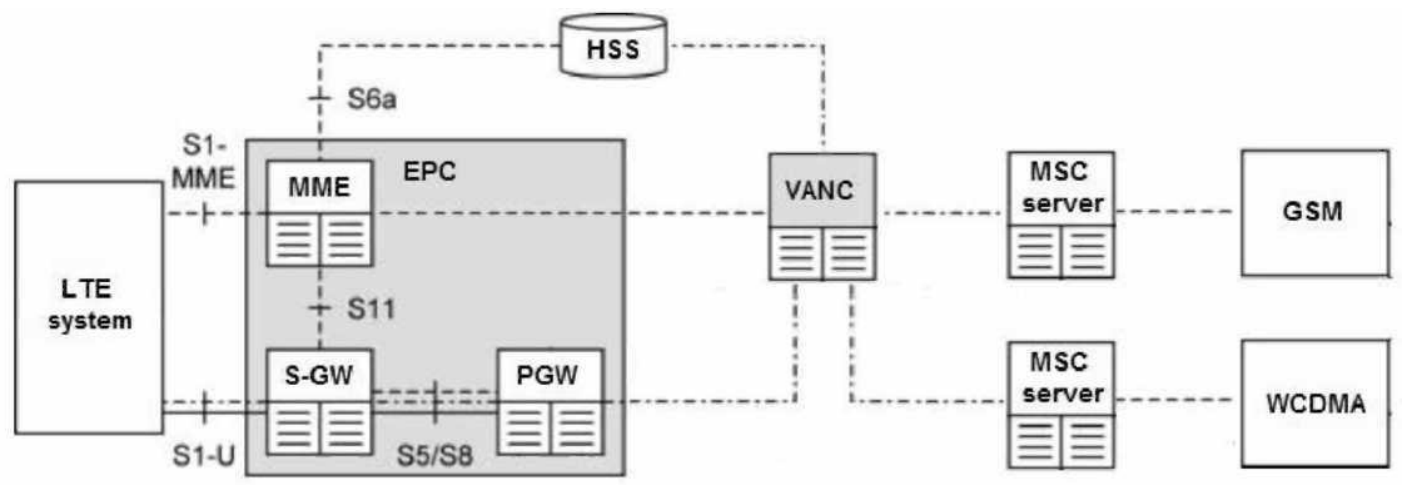

Figure 6: Voice over LTE via Generic Access system architecture

In comparison to the CS fallback technique, VoLGA offers a whole range of advantages. Since the data stream of a voice call is a normal packet data stream, the UE is not limited to only one connection, but can also use multiple connections simultaneously. More importantly, for this kind of communication, no fallback to GSM or WCDMA is required. A fallback is only issued in situations of LTE coverage loss, i.e. a continuation of the current voice call is realised through the packet domains of GSM or WCDMA.

\section{Additional Solutions}

Even though the previously mentioned approaches and techniques form a monopole that will most likely be implemented into the LTE system, several other possible solutions and additions have emerged. One of the most important additions to the IMS system and VoIP in LTE is a technique named Single Radio Voice Call Continuity (SRVCC). This functionality enables a seamless inter-system handover from VoIP services of the packet domain to the circuit switched domain in the event of coverage loss. The name "Single Radio" implies that the UE is not required to support dual-mode transmission, since the technique just 


\section{International Journal of Science and Research (IJSR) \\ ISSN (Online): 2319-7064}

Index Copernicus Value (2013): 6.14 | Impact Factor (2014): 5.611

affects the data stream. The main element in this architecture is the SR-VCC enhanced MSC server (i.e. S-IWF), which is an equivalent to the MSC server in CS fallback techniques. The S-IWF is based on already available CS core network components, requiring minimal software and hardware enhancements of the existing system. Its functions are the triggering of the SR-VCC handover procedure and the fallback process to GSM, UMTS or CDMA, as well as typical MSC functions such as connecting the voice call streams from one UE within LTE to the other UE in the CS network. To save pointless processing and keep the voice call latency low, the S-IWF is not included in the call structure if no handover is required.

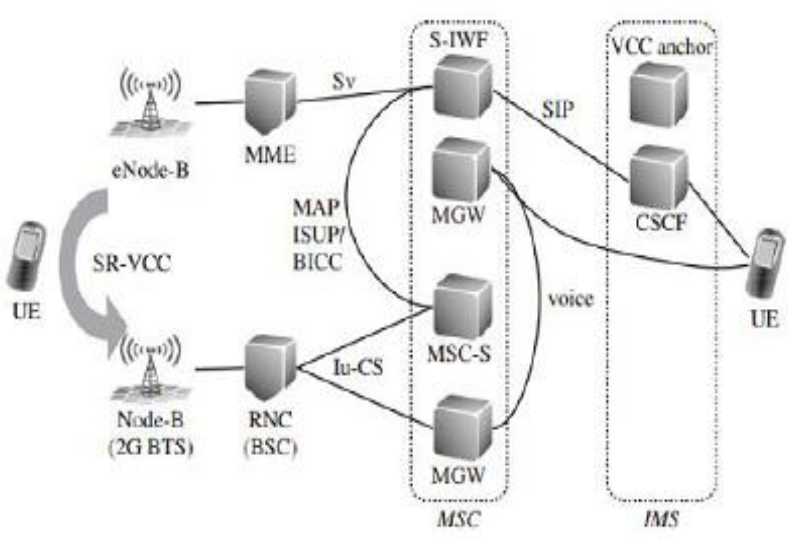

Figure 7: Block diagram of the SR-VCC architecture

The use of SR-VCC also enables the simultaneous use of voice and non-voice connections. The process which allows this type of multiplexing is carried out by the signal splitting functions in the MME. In cases of an inter-system handover, the non-voice transmission could get suppressed if the circuit switched target network does not support simultaneous voice and data functionality (e.g. GSM). The handover procedure for non-voice transmissions is carried out as for a normal inter-system handover. Additional information about the SR-VCC technology can be found in $[2]$.

Another technique that supports simultaneous transmission of voice and non-voice data is called Simultaneous Voice LTE (SV-LTE). The main difference to SR-VCC lies in the separate utilisation of multiple antennas, i.e. "Multiple Radio", which enables the UE to connect to both packet switched and circuit switched domain services. The SV-LTE concept is therefore a combination of the main two aspects mentioned above, providing the facilities of IMS and CS fallback at the same time. However, this advantage can also be seen as a disadvantage; since at least two antennas are used to support two different types of connection, the required processing is increased proportionally. The technique was therefore declared inefficient, as two active connections and twice the processing significantly impact the energy consumption of an UE.

\section{Problems and Challenges of Voice and Text Services in LTE}

As the above mentioned techniques evolve and slowly merge with the LTE system, their benefits but also their flaws are influencing a steady increasing number of UEs. To perfect these techniques, the following issues and flaws have to be addressed:

- Call preservation in events of coverage loss. This is a major problem in adaptations with third party VoIP providers, as no fallback function is applied. Its solution lies in the use of SR-VCC functionalities.

- Dual-mode transmission. Circuit switched fallback can only be used when the UE is within the coverage of both the LTE and GSM/WCDMA network. Otherwise, the attach procedure would fail, making the fallback and therefore the utilisation of voice calls impossible.

- Voice call latency. The delay during inter-system handover in circuit switched fallback can reach a few seconds, impacting the total delay budget.

- Fallback procedure. Since inter-system handovers represent one of the least reliable procedures in all of mobile communications, this issue results in a high number of dropped calls.

- Low network resiliency. This issue occurs when a MME connects to only one MSC. To solve this issue, support to add multiple MSC connection to the MME has to be provided, resulting in an improved network resiliency.

- SMS messaging via CS fallback. The sending of a large amount of messages would cause a large number of network reselections and handovers, rendering the service inefficient. This problem was solved by using the so called SMS over SGs technique.

\section{Conclusion}

Voice call and text messaging services still comprise most of a mobile operator's revenue, making the LTE implementation of these services a priority. Two main approaches have been introduced and applied in different adaptations: the use of packet related VoIP services and the utilisation of existing circuit switched networks through fallback techniques. As neither of these particular approaches provide all required features, different combinations of their adaptations are most likely to be standardised and used in the LTE system. Furthermore, this paper describes the specific additions and upgrades of the existing system architecture, as well as the techniques' problems and flaws.

\section{References}

[1] Cox, Christopher;"An Introduction to LTE - LTE, LTEAdvanced, SAE and 4G Mobile Communications", John Wiley \& Sons Ltd, Chichester, 2012.

[2] Holma, Harry; Toskala, Antti; "LTE for UMTS Evolution to LTE-Advanced", Second Edition, John Wiley \& Sons Ltd, Chichester, 2011.

[3] Dahlman, Erik; Parkvall, Stefan; Skold, Johan; Beming, Per; "3G Evolution - HSPA and LTE for Mobile Broadband", Second Edition, Academic Press by Elsevier, Oxford, 2008 


\section{International Journal of Science and Research (IJSR) \\ ISSN (Online): 2319-7064}

Index Copernicus Value (2013): 6.14 | Impact Factor (2014): 5.611

[4] Forsberg, Dan; Horn, Gunther; Moeller, Wolf-Dietrich; Niemi, Valtteri; "LTE Security", John Wiley \& Sons Ltd, Chichester, 2010

[5] "Overview of 3GPP Release 8", 3GPP, TR 21.101 and TR 41.101, Version 0.2.10, 2013

[6] "Overview of 3GPP Release 9", 3GPP, TR 21.101 and TR 41.101, Version 0.2.9, 2013

[7] "3GPP System Architecture Evolution (SAE); Security Architecture", ETSI, TS 33.401, Version 10.3.0, 2012

[8] Flore, Dino; "LTE RAN Architecture Aspects", 3GPP, Beijing, 2009.

[9] Myung, Hyung; Lim, Junsung; Goodman, David; „Single Carrier FDMA for Uplink Wireless Transmission", IEEE, New York, 2006

[10] Oestges, Claude; Clerckx, Bruno; "MIMO Wireless Communications - From Real-World Propagation to Space-Time Code Design", Academic Press by Elsevier, Oxford, 2007

[11] Kiziltan, Baran; Khan Majid; Velotti, Francesco; "Voice over IP - WLAN, 3G and LTE Issues", Chalmers University of Technology, Goteborg, 2011

[12] Bilogrevid, Igor; Jadliwala, Murtuza; Hubaux, JeanPierre; "Security Issues in Next Generation Mobile Networks: LTE and Femtocells", EPFL, Lausanne, 2010

[13] Orhanou, Ghizlane; El Hajji, Said; Bentaleb, Youssef; "SNOW 3G Stream Cipher Operation and Complexity Study", Universite Mohammed V Agdal, Rabat, 2010

[14] Rizzo, Carmine; Brookson, Charles; "Security for ICT the Work of ETSI", Fifth Edition, ETSI, Sophia Antipolis Cedex, 2013

[15] Poole,Ian;"Celular Telecoms",from the Internet,http://www.radioelectronics.com/info/cellulartelecomms. 